\title{
Tobacco use and related behaviors among staff and students in a university hospital: A large cross-sectional survey
}

Emmanuel Grolleau ${ }^{1,2}$, Véronique Fonteille ${ }^{3}$, Caroline Lebourgeois ${ }^{2,4}$, Marie Darrason ${ }^{1}$, Philippe Miche ${ }^{2,5}$, Delphine Ragon$n^{n} t^{6}$, Gilles Freyer ${ }^{7}$, Amélie Deculty ${ }^{8}$, Carine Gippet ${ }^{9}$, Céline Leclercq ${ }^{10}$, Carole Neugnot ${ }^{11}$, Radoudja Malek ${ }^{12}$, Odile Perdriolat $^{13}$, Michele Rigaud ${ }^{12}$, Séverine Torrecillas ${ }^{1,8}$, Maud-Catherine Barral' ${ }^{13}$, Pierre-Jean Souquet' ${ }^{1}$, Jean-Baptiste Fassier ${ }^{14}$, Lénaïck Tanguy ${ }^{7,15}$, Benjamin Rolland ${ }^{3,6,16}$, Sébastien Couraud ${ }^{1,17}$, for the Hospices Civils de Lyon COLT committee

\section{ABSTRACT}

INTRODUCTION Smoking prevalence in the overall population in France was $27 \%$ in 2017 . There are few data about smoking prevalence in hospital workers. The aim of this study was to assess prevalence of current smoking in student and staff populations at Lyon University Hospital. Secondary objectives were to identify main variables associated with current smoking and willingness to quit.

METHODS We designed a single center, cross-sectional survey, using printed questionnaires. During one day, all registered staff and students were surveyed. We used optical reading to extract information from questionnaires. We performed univariate and multivariate analysis adjusted on most relevant factors.

RESULTS We analyzed 9712 questionnaires. The participating rates were high: $40.6 \%$ in the student cohort and $51.5 \%$ in the staff cohort. The proportion of current cigarette users was $26 \%$ in students and $25 \%$ in staff. In multivariate analysis, current smoking was significantly associated with: younger age, male sex, occupation type (e.g. logistical staff, and paramedical students), overnight work, and e-cigarette use. Among smokers, 53\% reported a willingness to quit. In multivariate analysis, number of quit attempts, and feeling symptoms from tobacco were associated with willingness to quit.

conclusions Current smoking is less frequent in our cohorts of hospital staff and students than in the general French population. However, there are deep disparities in current smoking prevalence underlining a heterogeneous population. Among smokers, the majority reported a willingness to quit and some predictive factors may help to target this audience.

\section{AFFILIATION}

1 Service de Pneumologie Aigue Spécialisée et Cancérologie Thoracique, Centre hospitalier Lyon Sud, Hospices Civils de Lyon, Pierre Bénite, France

2 Faculté de Médecine Lyon Est, Université Claude Bernard Lyon 1, Lyon, France

3 Equipe de Liaison en Addictologie, Groupement Hospitalier Est, Hospices Civils de Lyon, Bron,

France

4 Direction de la Performance et du Contrôle de Gestion, Hospices Civils de Lyon, Lyon, France 5 Direction de I'Organisation, de la Qualité des Soins et des Relations avec les Usagers Hospices Civils de Lyon, Lyon, France

6 Service d'Addictologie, Hôpital Edouard Herriot, Hospices Civils de Lyon, Lyon, France 7 Institut de Cancérologie des Hospices Civils de Lyon, Hospices Civils de Lyon, Lyon, France 8 Centre de Coordination en Cancérologie, Groupement Hospitalier Sud, Hospices Civils de Lyon, Pierre Bénite, France

9 Centre de Coordination en Cancérologie, Hospices Civils de Lyon, Lyon, France 10 Centre de Coordination en Cancérologie, Groupement Hospitalier Nord, Hospices Civils de Lyon, Lyon, France

11 Centre de Coordination en Cancérologie, Groupement Hospitalier Est, Hospices Civils de Lyon, Bron, France

12 Centre de Coordination en Cancérologie, Groupement Hospitalier Centre, Hospices Civils de Lyon, Lyon, France

13 Unité de Tabacologie, Service de Maternité, Hôpital Femme-Mère Enfant, Hospices Civils de Lyon, Bron, France

14 Service de Médecine et Santé au Travail, Centre Hospitalier Lyon Sud, Hospices Civils de Lyon, Pierre Bénite, France

15 Direction de la Coopération et des Stratégies, Hospices Civils de Lyon, Lyon, France

16 Service Universitaire d'Addictologie de Lyon, Centre Hospitalier du Vinatier, Bron, France 17 Equipe IBISCUS, Centre d'Innovation en Cancérologie de Lyon (CICLY), Faculté de Médecine Lyon Sud, Oullins, France 


\begin{abstract}
CORRESPONDENCE TO
Sébastien Couraud. Service de Pneumologie Aigue Spécialisée et Cancérologie Thoracique Centre Hospitalier Lyon Sud, Hospices Civils de Lyon, 165 Chemin du grand Revoyet, 69310 Pierre bénite, France. E-mail: sebastien. couraud@chu-lyon.fr
\end{abstract}

\section{KEYWORDS}

tobacco, caregiver, medical student, e-cigarette, tobacco quitting

Received: 3 March 2021

Revised: 14 April 2021

Accepted: 18 May 2021

\section{INTRODUCTION}

Tobacco use is the second most preventable factor of mortality in the world, and the first in France ${ }^{1,2}$. Five million deaths each year are attributable to smoking, and it could reach 10 million deaths per year in 2030 worldwide $^{1}$. In France, smoking prevalence is monitored at a national level through epidemiological studies conducted since 1992 (health barometer, Santé Publique France). Smoking prevalence in France was stable between 2010 and 2016 and started to decrease in 2017. Healthcare professionals and healthcare students are a particular category of the population, insofar as they are supposed to be educated about smoking dangers. Of course, working conditions in healthcare settings can be stressful, and thus foster tobacco smoking. But, overall, the literature about smoking prevalence among hospital staff and students compared with the general population is controversial with some studies showing a higher prevalence and some a lower ${ }^{3-6}$. Nevertheless, prevention in those populations looks decisive, not only for their selfhealth but also to contribute to patient smoking prevention; indeed, health professionals who consume tobacco themselves are often less likely to engage in tobacco control than their non-tobacco-using counterparts ${ }^{7}$.

The Lyon University Hospital (Hospices Civils de Lyon) is the second biggest French university hospital. It has 23000 employees, representing more than 170 occupations in 14 different sites. It also integrates two medical faculties and 6 paramedical schools. Since 2017, the Hospices Civils de Lyon initiated a large tobacco smoking control initiative: the COLT committee (Comité de Lutte contre le Tabac) which gathers preventive actions against tobacco smoking, and provides staff and patients with help for smoking cessation.

The aim of this study was to estimate the prevalence of smoking among student and staff cohorts in the Lyon University Hospital. Secondary objectives were to explore the main variables associated with tobacco smoking and willingness to quit in these two categories of population. This study should help to identify various factors for improving prevention and action on tobacco smoking cessation.

\section{METHODS}

\section{General design and objectives}

We designed a cross-sectional survey conducted at a pre-specified date, by paper questionnaires (anonymous responses), in two cohorts: students and staff at Lyon University Hospital. The day of the survey was set at Thursday, 23 November 2017. There were 8314 students and 13723 staff attending work or faculty on the day of the survey.

The main objective of this study was to assess prevalence of current smoking in each cohort. Secondary objectives were to investigate main variables associated with current smoking among the overall population; main variables were associated with willingness to quit in the current smoker population.

The study was announced to staff and students some days in advance as a key-action during the Mois sans Tabac program (a special quitting program launched by the French Ministry of Health each year in November). Questionnaires were distributed in each unit of the hospital through the network of proximity managers. Staff returned the questionnaire in an urn or envelop, and all questionnaires from the same unit were thus returned to the secretary of the study. 
Overnight distribution to night-staff was also planned and delivered in a similar manner. Student questionnaires were distributed by administrative staff in each faculty or paramedical school, directly to students during courses of the day, and returned by the same way. All questionnaires were then treated by optical reading. Overall, this study was conducted under the STROBE criteria ${ }^{8}$.

\section{Eligibility criteria \\ Staff}

Each individual at work on the day or the night of the survey, and regularly registered as a staff of Hospices Civils de Lyon, whatever their occupation or type of contract (temporary or permanent), and who agreed to participate. Interim staff were excluded.

\section{Students}

Each individual who was registered as a regular student at one of the medical faculty/paramedical schools of Hospices Civils de Lyon, in initial training, and on site the day of the survey, and who agreed to participate.

\section{Questionnaires}

Each questionnaire had a ticking box at the beginning allowing the individual to refuse participation in the study. Participants who ticked this box were excluded from the entire analysis. In addition, most of questions allowed a 'refusal' answer. Refusal answers and missing values were reported as such in the descriptive tables. They were then removed in other analysis.

There were two different questionnaires, one for each cohort. Questionnaires for staff and students were very similar (Supplementary file). Questions about sociodemographics (gender, age, presence of a pregnant woman and/or young child at home), smoking status, number of quit attempts, willingness to quit, tobacco-related symptoms, and e-cigarette use, were identical in the questionnaires. A main difference in the questionnaires was about: occupation details (including overnight work and contract type) in staff; focused on studies (faculty/ school and level) in students; and using French Statistics Agency categories for patients. In staff, we also question them about their administrative category [A (highest), B (intermediate) and C (lowest)] which is commonly used in French administration to classify workers depending on their level of diploma. Three additional questions were proposed to both staff and students: whether someone already talk about quitting at work/school; whether they smoke most at home or at work/school; as well as their opinion whether their study/work worsened their dependence.

Questionnaires were edited and printed for automatic optical reading. For this reason, we used only categorical variables. For instance, age was presented in categories for this reason.

\section{Smoking status definition}

Current smokers were defined as individuals claiming to currently smoke or who quit since less than one year ago. Never smokers were defined as individuals who reported having smoked less than 100 cigarettes during their lifetime ${ }^{9}$. Former smokers were defined as individuals who smoked more than 100 cigarettes in their lifetime and who quit for at least 12 months. A similar definition was used for e-cigarette users. Individuals who used at least one bottle of e-liquid in their lifetime were considered as ever users. Individuals who did not use e-cigarettes for at least one year were considered as former users; while others were considered as current users.

\section{Statistics analysis}

Categorical variables were expressed as percent and compared using the chi-squared test. We used binary logistic regression (enter method) with variable showing significant difference in univariate analysis, and without missing value (threshold set up at $5 \%$ ). There was no continuous variable (not readable by optic reading machine). All tests were two-sided.

To take into account uncertainty due to multiple testing, we applied a Bonferroni correction. The level of significance was thus set to $\mathrm{p}<0.0017$.

\section{Ethics}

Participating in the study was systematically proposed but participants were free not to participate. An information sheet (approved by the ethics committee) was available at the reverse of each questionnaire. All responses were anonymous.

This study was sponsored by Hospices Civils de 
Lyon under the reference 69HCL17_0481, and was accepted by the Sud-Ouest et Outre Mer III ethics committee (Comité de Protection des Personnes) under the reference 2017-A02168-45 on 25 October 2017. It was declared to the local correspondent from French Informatics and Freedom Agency under the reference MR0003-17-153. It was registered in clinicaltrials.gov under the reference NCT03268980. The study (and questionnaire) was also approved by physicians and staff representatives, as well as school and university managers.

\section{RESULTS}

\section{Main demographics of the cohorts}

Overall, 22037 individuals were surveyed (8314 students and 13723 staff) and 9712 questionnaires were analyzed (3325 from the student sample, and
6387 from the staff sample). Thus, the participating rates were $40.6 \%$ in the student cohort and $51.5 \%$ in the staff cohort (Figure 1).

The main descriptive data of the two samples are presented in Table 1 . A vast majority of participant were female ( $72 \%$ in student and $78 \%$ in staff). Most of students were from medical schools (77\%). In staff, main occupations were paramedical (59\%), medical (16\%), administrative (16\%), and other. The vast majority of respondents in staff were permanent workers $(78 \%)$ with no overnight work $(80 \%)$. Overall, most of student and staff self-estimated themselves as well informed about tobacco (87\% of students and $84 \%$ of staff). The proportion of current cigarettes users was $26 \%$ in students and $25 \%$ in staff; while the proportion of former smokers was $6 \%$ in students and $20 \%$ in staff $\left(\mathrm{p}<10^{-4}\right)$. The proportion

Figure 1. Flow chart of the study

\section{Student cohort}

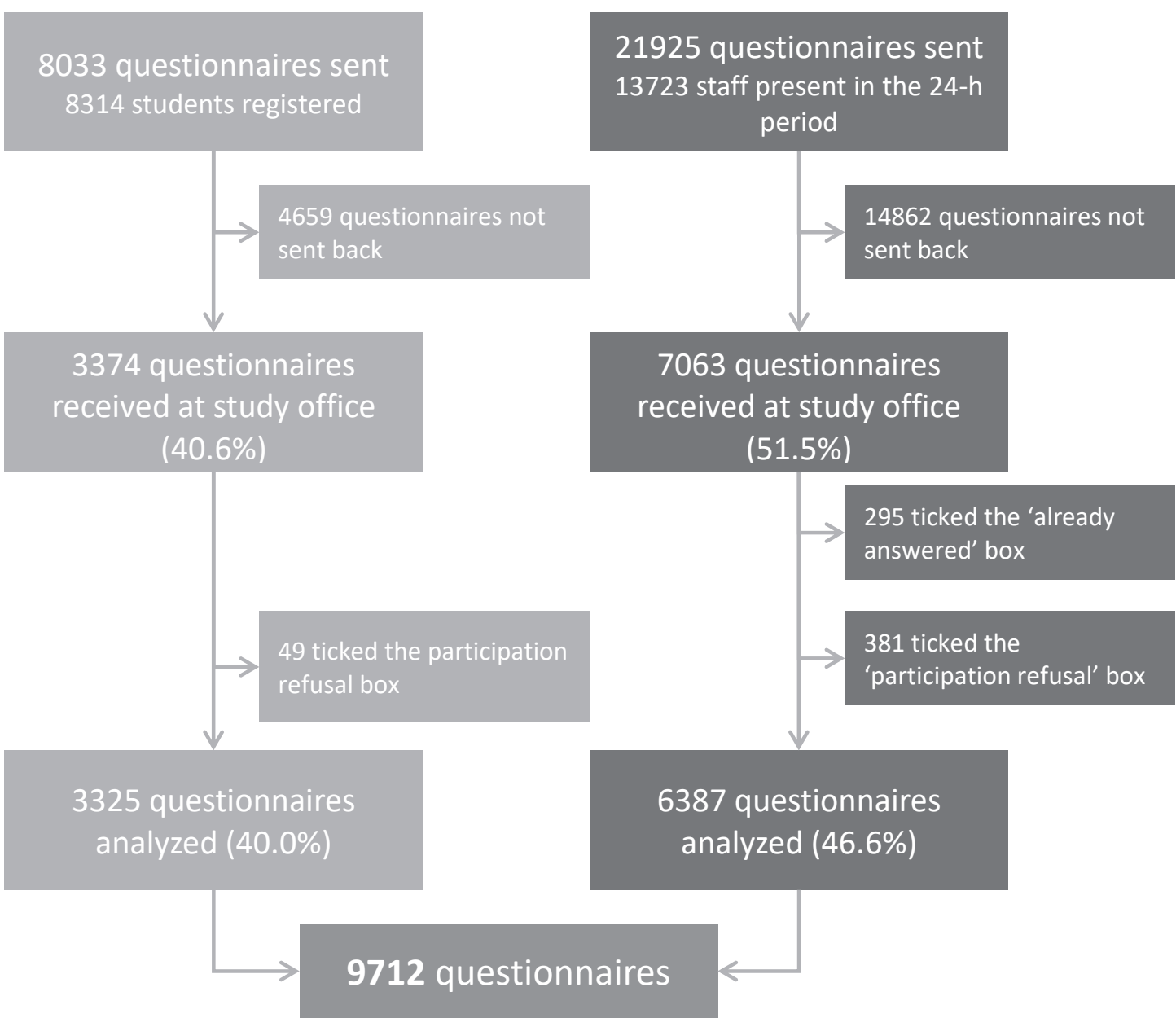

Staff cohort

14862 questionnaires not sent back

7063 questionnaires

295 ticked the 'already

answered' box

381 ticked the

'participation refusal' box 
Table 1. Description of the student and staff cohorts ( $\mathrm{N}=9712)$

\begin{tabular}{|c|c|c|c|c|c|c|c|c|}
\hline \multirow[t]{2}{*}{ Variable } & \multirow[t]{2}{*}{ Category } & \multicolumn{2}{|c|}{$\begin{array}{c}\text { Students } \\
(\mathbf{N}=3325)\end{array}$} & \multicolumn{2}{|c|}{$\begin{array}{c}\text { Staff } \\
(\mathrm{N}=6387)\end{array}$} & \multirow[b]{2}{*}{$p$} & \multicolumn{2}{|c|}{ Total } \\
\hline & & $n$ & $\%$ & $n$ & $\%$ & & $n$ & $\%$ \\
\hline \multirow[t]{3}{*}{ Gender } & Female & 2378 & 71.9 & 4898 & 78.8 & \multirow[t]{3}{*}{$<10^{-4}$} & 7276 & 76.4 \\
\hline & Male & 929 & 28.1 & 1319 & 21.2 & & 2248 & 23.6 \\
\hline & Missing & 18 & & 170 & & & 188 & \\
\hline \multirow[t]{10}{*}{ Age (years) } & $<15$ & 3 & 0.1 & 1 & 0.0 & \multirow[t]{10}{*}{$\mathrm{NC}$} & 4 & 0.0 \\
\hline & $15-19$ & 1858 & 56.3 & 22 & 0.3 & & 1880 & 19.4 \\
\hline & $20-25$ & 1108 & 33.5 & 679 & 6.1 & & 1787 & 11.4 \\
\hline & $26-34$ & 203 & 6.1 & 1550 & 13.9 & & 1753 & 12.0 \\
\hline & $35-44$ & 90 & 2.7 & 1411 & 14.7 & & 1501 & 11.6 \\
\hline & $45-54$ & 39 & 1.2 & 1556 & 17.1 & & 1595 & 12.8 \\
\hline & $55-64$ & 1 & 0.0 & 979 & 11.5 & & 980 & 6.8 \\
\hline & $65-75$ & 0 & 0.0 & 23 & 0.2 & & 23 & 0.1 \\
\hline & $>75$ & 1 & 0.0 & 0 & 0.0 & & 1 & 0.0 \\
\hline & Missing & 22 & & 166 & & & 188 & \\
\hline \multirow{4}{*}{$\begin{array}{l}\text { Pregnant woman } \\
\text { and/or young child } \\
\text { (<2 years) at home }\end{array}$} & No & 3027 & 96.6 & 4852 & 89.2 & \multirow[t]{4}{*}{$<10^{-4}$} & 7879 & 91.9 \\
\hline & Yes $(\geq 1)$ & 108 & 3.4 & 590 & 10.8 & & 698 & 8.1 \\
\hline & Refusal & 14 & & 24 & & & 38 & \\
\hline & Missing & 176 & & 921 & & & 1097 & \\
\hline \multirow[t]{12}{*}{ Occupation } & Medical & 2534 & 77.2 & 988 & 16.0 & \multirow[t]{12}{*}{ NC } & 3522 & 37.3 \\
\hline & Paramedical & 749 & 22.8 & 3659 & 59.3 & & 4408 & 46.6 \\
\hline & Nurse's school & 507 & 15.4 & & & & 507 & 5.4 \\
\hline & Nurse manager's school & 58 & 1.8 & & & & 58 & 0.6 \\
\hline & Radiology technician's school & 76 & 2.3 & & & & 76 & 0.8 \\
\hline & Other paramedical schools & 108 & 3.3 & & & & 108 & 1.1 \\
\hline & Non-caregiver & & & 1522 & 24.7 & & 1522 & 16.1 \\
\hline & Technical/security & & & 276 & 4.5 & & 276 & 2.9 \\
\hline & Administrative & & & 955 & 15.5 & & 955 & 10.1 \\
\hline & Logistical & & & 185 & 3.0 & & 185 & 2.0 \\
\hline & Scientific & & & 106 & 1.7 & & 106 & 1.1 \\
\hline & Missing & 42 & & 1740 & & & 1782 & \\
\hline \multirow{5}{*}{$\begin{array}{l}\text { Staff } \\
\text { administrative } \\
\text { category }\end{array}$} & $A$ & & & 2473 & 48.8 & \multirow[t]{5}{*}{$\mathrm{NC}$} & 2473 & 48.8 \\
\hline & B & & & 1458 & 28.7 & & 1458 & 28.7 \\
\hline & C & & & 1141 & 22.5 & & 1141 & 22.5 \\
\hline & Unknown & & & 434 & & & 434 & \\
\hline & Missing & & & 881 & & & & \\
\hline \multirow{4}{*}{$\begin{array}{l}\text { Self-estimate } \\
\text { as being well } \\
\text { informed on } \\
\text { tobacco }\end{array}$} & No & 408 & 13.1 & 982 & 16.5 & \multirow[t]{4}{*}{$<10^{-4}$} & 1390 & 15.4 \\
\hline & Yes & 2703 & 86.9 & 4962 & 83.5 & & 7665 & 84.6 \\
\hline & Refusal & 101 & & 253 & & & 354 & \\
\hline & Missing & 113 & & 190 & & & 303 & \\
\hline \multirow[t]{5}{*}{ Staff contract } & Student & 3325 & 100.0 & & & \multirow[t]{5}{*}{$\mathrm{NC}$} & 3325 & 37.0 \\
\hline & Temporary & & & 1249 & 22.1 & & 1249 & 13.9 \\
\hline & Permanent & & & 4404 & 77.9 & & 4404 & 49.1 \\
\hline & Refusal & & & 71 & & & 71 & \\
\hline & Missing & & & 663 & & & 663 & \\
\hline
\end{tabular}


Table 1. Continued

\begin{tabular}{|c|c|c|c|c|c|c|c|c|}
\hline \multirow[t]{2}{*}{ Variable } & \multirow[t]{2}{*}{ Category } & \multicolumn{2}{|c|}{$\begin{array}{l}\text { Students } \\
(\mathbf{N}=3325)\end{array}$} & \multicolumn{2}{|c|}{$\begin{array}{c}\text { Staff } \\
(\mathbf{N}=6387)\end{array}$} & \multirow[b]{2}{*}{$p$} & \multicolumn{2}{|c|}{ Total } \\
\hline & & $n$ & $\%$ & $n$ & $\%$ & & $n$ & $\%$ \\
\hline \multirow[t]{4}{*}{ Overnight work } & Yes & & & 1252 & 20.4 & \multirow[t]{4}{*}{$\mathrm{NC}$} & & \\
\hline & No & & & 4893 & 79.6 & & & \\
\hline & Refusal & & & 15 & & & & \\
\hline & Missing & & & 227 & & & & \\
\hline \multirow[t]{4}{*}{ Cigarette user } & Never & 2267 & 68.7 & 3480 & 55.3 & \multirow[t]{4}{*}{$<10^{-4}$} & 5747 & 59.9 \\
\hline & Former & 184 & 5.6 & 1270 & 20.2 & & 1454 & 15.1 \\
\hline & Current & 851 & 25.8 & 1548 & 24.6 & & 2399 & 25.0 \\
\hline & Missing & 23 & & 89 & & & 112 & \\
\hline \multirow[t]{4}{*}{ E-cigarette user } & Never & 2908 & 90.5 & 4966 & 87.1 & \multirow[t]{4}{*}{$<10^{-4}$} & 7874 & 88.3 \\
\hline & Current & 161 & 5.0 & 403 & 7.1 & & 564 & 6.3 \\
\hline & Former & 144 & 4.5 & 333 & 5.8 & & 477 & 5.4 \\
\hline & Missing & 112 & & 685 & & & 797 & \\
\hline
\end{tabular}

NC: Not calculated. Percentages exclude missing and refusal.

of ever user of e-cigarettes was $10 \%$ in students and $13 \%$ in staff $\left(\mathrm{p}<10^{-4}\right)$.

\section{Being a current smoker}

Table 2 shows the main characteristics of current smokers in the two cohorts. The majority $(82 \%$ of students and $67 \%$ of staff) reported smoking less than 10 cigarettes per day. Overall, only $34 \%$ ( $42 \%$ among students and 30\% among staff) reported that they never tried to quit smoking, and $53 \%$ had a willingness to quit. Around one-third (31\%) currently felt symptoms attributed to tobacco smoking. Around $28 \%$ only, indicated having received a proposal to quit during their work/studies; while $72 \%$ of students and $42 \%$ of staff self-estimated that work/study increases their dependence. About $64 \%$ of students underlined being more prone to smoke at school/faculty than at home.

In the Supplementary file (Material 3), we tried to identify predictive variables associated with current smoker status compared to non-smoker status (former and never smoker together). The proportion of male current smokers was slightly higher in staff but not in students. In the staff population, age was significantly associated with current smoking, youngest categories being associated with the highest prevalence of current smoking. Occupation was also strongly associated with current smoking.
E-cigarette use was strongly associated with current smoking in students $(84.5 \%)$.

In multivariable analysis most of these variables remained significantly associated with current smoking (Table 3). Male gender was associated with an increased risk of being a current smoker $\left(\mathrm{AOR}=1.27 ; 95 \%\right.$ CI: $\left.1.123-1.432, \mathrm{p}<10^{-4}\right)$ but not in the student cohort alone. Being aged $<25$ years was associated with higher smoking prevalence (AOR=1.67; 95\% CI: 1.38-2.02, $\left.\mathrm{p}<10^{-4}\right)$ compared to the 35-44 years reference category. By contrast, belonging to the $45-54$ years category was associated with a lower risk $(\mathrm{AOR}=0.74 ; 95 \%$ CI: $0.62-0.89$, $\left.\mathrm{p}<10^{-4}\right)$. Night work was associated with current smoking $\left(\mathrm{AOR}=1.46 ; 95 \% \mathrm{CI}\right.$ : $\left.1.25-1.70, \mathrm{p}<10^{-4}\right)$; while self-estimate of being informed about smoking was associated with a lower risk overall $(\mathrm{AOR}=0.68$; 95\% CI: $\left.0.59-0.78, \mathrm{p}<10^{-4}\right)$. Finally, compared to administrative staff, logistical staff had higher risk of being current smokers (AOR=2.32; 95\% CI: 1.61 $\left.3.35, \mathrm{p}<10^{-4}\right)$. Medical staff and medical students had a significantly decreased risk $(A O R=0.53 ; 95 \%$ CI: $0.42-0.69, \mathrm{p}<10^{-4}$ and AOR $=0.49 ; 95 \%$ CI: $0.38-$ $0.62, \mathrm{p}<10^{-4}$; respectively).

\section{Becoming a quitter}

Supplemental file (Material 4) gives data on willingness to quit smoking among current smokers in 
both cohorts, and factors associated with willingness to quit. Age, occupation, overnight work, quit attempts, and feeling tobacco-related symptoms, were strongly associated with willingness to quit.
Results of the multivariate analysis in this population are shown in Table 4. The model comprises 1536 individuals (on 1850) and 8 variables. The strongest factors associated with

Table 2. Current smoker's main characteristics

\begin{tabular}{|c|c|c|c|c|c|c|c|}
\hline \multirow[t]{2}{*}{ Variable } & \multirow[t]{2}{*}{ Category } & \multicolumn{2}{|c|}{$\begin{array}{l}\text { Students } \\
(\mathbf{N}=851)\end{array}$} & \multicolumn{2}{|c|}{$\begin{array}{c}\text { Staff } \\
(\mathrm{N}=1548)\end{array}$} & \multicolumn{2}{|c|}{$\begin{array}{c}\text { Total } \\
(\mathbf{N}=2399)\end{array}$} \\
\hline & & $n$ & $\%$ & $n$ & $\%$ & $n$ & $\%$ \\
\hline \multirow{4}{*}{$\begin{array}{l}\text { Number of cigarettes smoked } \\
\text { during life (mean) }\end{array}$} & $<10$ & 646 & 82.0 & 1000 & 67.0 & 1646 & 72.2 \\
\hline & $10-20$ & 129 & 16.4 & 430 & 28.8 & 559 & 24.5 \\
\hline & $>20$ & 13 & 1.6 & 63 & 4.2 & 76 & 3.3 \\
\hline & Missing & 63 & & 55 & & 118 & \\
\hline \multirow[t]{6}{*}{ Previous quit attempts } & 0 & 278 & 41.6 & 372 & 29.7 & 650 & 33.9 \\
\hline & 1 & 161 & 24.1 & 312 & 24.9 & 473 & 24.6 \\
\hline & 2 & 104 & 15.6 & 219 & 17.5 & 323 & 16.8 \\
\hline & $>2$ & 125 & 18.7 & 348 & 27.8 & 473 & 24.6 \\
\hline & Refusal & 9 & & 0 & & 9 & \\
\hline & Missing & 174 & & 297 & & 471 & \\
\hline \multirow[t]{4}{*}{ Willing to quit } & No & 353 & 53.0 & 515 & 43.5 & 868 & 46.9 \\
\hline & Yes & 313 & 47.0 & 669 & 56.5 & 982 & 53.1 \\
\hline & Refusal & 29 & & 81 & & 110 & \\
\hline & Missing & 156 & & 283 & & 439 & \\
\hline \multirow{4}{*}{$\begin{array}{l}\text { Current feeling of symptoms } \\
\text { from smoking (self-estimated) }\end{array}$} & No & 500 & 69.1 & 909 & 69.8 & 1409 & 69.5 \\
\hline & Yes & 224 & 30.9 & 394 & 30.2 & 618 & 30.5 \\
\hline & Refusal & 14 & & 32 & & 46 & \\
\hline & Missing & 113 & & 213 & & 326 & \\
\hline \multirow{6}{*}{$\begin{array}{l}\text { Has someone at work/school } \\
\text { already proposed for you to } \\
\text { quit? }\end{array}$} & No & 539 & 72.6 & 387 & 70.4 & 926 & 71.7 \\
\hline & Yes work/university physician & 30 & 4.0 & 73 & 13.3 & 103 & 8.0 \\
\hline & Yes GP & 36 & 4.9 & 21 & 3.8 & 57 & 4.4 \\
\hline & Yes colleagues/ trainers & 137 & 18.5 & 69 & 12.5 & 206 & 15.9 \\
\hline & Refusal & 8 & & 22 & & 30 & \\
\hline & Missing & 101 & & 976 & & 1077 & \\
\hline \multirow{4}{*}{$\begin{array}{l}\text { Do you consider that work/ } \\
\text { training increases cigarette } \\
\text { smoking for you? }\end{array}$} & No & 214 & 27.6 & 320 & 57.8 & 534 & 40.2 \\
\hline & Yes & 561 & 72.4 & 234 & 42.2 & 795 & 59.8 \\
\hline & Refusal & 9 & & 25 & & 34 & \\
\hline & Missing & 67 & & 969 & & 1036 & \\
\hline \multirow[t]{5}{*}{ Where do you smoke more? } & Work/school/faculty & 487 & 64.3 & 143 & 34.9 & 630 & 54.0 \\
\hline & Home & 128 & 16.9 & 153 & 37.3 & 281 & 24.1 \\
\hline & Equally everywhere & 142 & 18.8 & 114 & 27.8 & 256 & 21.9 \\
\hline & Refusal & 10 & & 45 & & 55 & \\
\hline & Missing & 84 & & 1093 & & 1177 & \\
\hline \multirow[t]{4}{*}{ E-cigarette user status } & Never & 587 & 69.6 & 1147 & 84.6 & 1734 & 78.9 \\
\hline & Current & 135 & 16.0 & 136 & 10.0 & 271 & 12.3 \\
\hline & Former (>1 year) & 121 & 14.4 & 72 & 5.3 & 193 & 8.8 \\
\hline & Missing & 8 & & 193 & & 201 & \\
\hline
\end{tabular}

Percentages exclude missing and refusal. 
Research Paper

Table 3. Multivariate analysis of risk of current smoking in the student and staff cohorts and overall

\begin{tabular}{|c|c|c|c|c|c|c|c|c|c|c|c|c|c|c|c|c|}
\hline \multirow[t]{3}{*}{ Variable } & \multirow[t]{3}{*}{ Category } & \multicolumn{5}{|c|}{ Staff cohort $(\mathrm{N}=6387)$} & \multicolumn{5}{|c|}{ Student cohort $(\mathrm{N}=3325)$} & \multicolumn{5}{|c|}{$\operatorname{ALL}(\mathbf{N}=9712)$} \\
\hline & & \multirow{2}{*}{$\begin{array}{l}N=5579 \\
\text { in the } \\
\text { model }\end{array}$} & \multirow[t]{2}{*}{ AOR } & \multicolumn{2}{|c|}{$95 \%$ CI } & \multirow[t]{2}{*}{$p$} & \multirow{2}{*}{$\begin{array}{l}\mathrm{N}=3125 \\
\text { in the } \\
\text { model }\end{array}$} & \multirow[t]{2}{*}{ AOR } & \multicolumn{2}{|c|}{$95 \%$ CI } & \multirow[t]{2}{*}{$p$} & \multirow{2}{*}{$\begin{array}{l}\mathrm{N}=8704 \\
\text { in the } \\
\text { model }\end{array}$} & \multirow[t]{2}{*}{ AOR } & \multicolumn{2}{|c|}{$95 \% \mathrm{CI}$} & \multirow[t]{2}{*}{$p$} \\
\hline & & & & Lower & Upper & & & & Lower & Upper & & & & Lower & Upper & \\
\hline \multirow[t]{2}{*}{ Gender } & Female & 4407 & 1 & & & & 2240 & 1 & & & & 6647 & 1 & & & \\
\hline & Male & 1172 & 1.376 & 1.171 & 1.617 & $<10^{-4}$ & 885 & 1.160 & 0.964 & 1.396 & 0.116 & 2057 & 1.269 & 1.123 & 1.432 & $<10^{-4}$ \\
\hline \multirow[t]{5}{*}{ Age (years) } & $\leq 25$ & 623 & 1.629 & 1.315 & 2.018 & $<10^{-4}$ & 2826 & 1.562 & 0.928 & 2.628 & 0.093 & 3449 & 1.668 & 1.381 & 2.016 & $<10^{-4}$ \\
\hline & $26-34$ & 1423 & 1.199 & 1.006 & 1.429 & 0.042 & 189 & 0.904 & 0.497 & 1.646 & 0.742 & 1612 & 1.162 & 0.983 & 1.375 & 0.079 \\
\hline & $35-44$ & 1269 & 1 & & & & 75 & 1 & & & & 1344 & 1 & & & \\
\hline & $45-54$ & 1381 & 0.747 & 0.622 & 0.899 & 0.002 & 34 & 0.685 & 0.269 & 1.745 & 0.427 & 1415 & 0.743 & 0.621 & 0.890 & 0.001 \\
\hline & $\geq 55$ & 883 & 0.496 & 0.394 & 0.625 & $<10^{-4}$ & 1 & 0.000 & 0.000 & & 1.000 & 884 & 0.494 & 0.393 & 0.621 & $<10^{-4}$ \\
\hline \multirow[t]{3}{*}{ Overnight work } & No & 4409 & 1 & & & & 0 & & & & & 4409 & 1 & & & \\
\hline & yes & 1170 & 1.453 & 1.243 & 1.699 & $<10^{-4}$ & 0 & & & & & 1170 & 1.456 & 1.246 & 1.702 & $<10^{-4}$ \\
\hline & Student & 0 & & & & & 3125 & & & & & 3125 & $\mathrm{NC}$ & & & \\
\hline \multirow[t]{8}{*}{ Occupation } & Administrative & 855 & 1 & & & & 0 & & & & & 855 & 1 & & & \\
\hline & Technical/security & 246 & 1.005 & 0.713 & 1.417 & 0.977 & 0 & & & & & 246 & 1.049 & 0.748 & 1.470 & 0.782 \\
\hline & Logistical & 160 & 2.214 & 1.528 & 3.209 & $<10^{-4}$ & 0 & & & & & 160 & 2.324 & 1.613 & 3.349 & $<10^{-4}$ \\
\hline & Scientific & 102 & 0.746 & 0.449 & 1.238 & 0.256 & 0 & & & & & 102 & 0.748 & 0.451 & 1.242 & 0.262 \\
\hline & Medical & 909 & 0.531 & 0.411 & 0.687 & $<10^{-4}$ & 0 & & & & & 909 & 0.537 & 0.417 & 0.693 & $<10^{-4}$ \\
\hline & Paramedical & 3307 & 1.010 & 0.838 & 1.218 & 0.914 & 0 & & & & & 3307 & 1.006 & 0.835 & 1.212 & 0.950 \\
\hline & Medical student & 0 & & & & & 2407 & 1 & & & & 2407 & 0.489 & 0.383 & 0.624 & $<10^{-4}$ \\
\hline & Paramedical student & 0 & & & & & 718 & 2.904 & 2.374 & 3.553 & $<10^{-4}$ & 718 & 1.420 & 1.104 & 1.826 & 0.006 \\
\hline \multirow{3}{*}{$\begin{array}{l}\text { Self-estimate as } \\
\text { being well informed } \\
\text { on tobacco }\end{array}$} & No & 883 & 1 & & & & 397 & 1 & & & & 1280 & 1 & & & \\
\hline & Yes & 4484 & 0.683 & 0.578 & 0.807 & $<10^{-4}$ & 2630 & 0.676 & 0.533 & 0.858 & 0.001 & 7114 & 0.680 & 0.594 & 0.780 & $<10^{-4}$ \\
\hline & Refusal & 212 & 1.335 & 0.964 & 1.849 & 0.081 & 98 & 0.893 & 0.542 & 1.474 & 0.659 & 310 & 1.166 & 0.889 & 1.530 & 0.267 \\
\hline
\end{tabular}

AOR: adjusted odds ratio. NC: Not calculated (redundant variable). 
Research Paper

Table 4. Multivariate analysis of willingness to quit among current smokers in the student and staff cohorts and overall

\begin{tabular}{|c|c|c|c|c|c|c|c|c|c|c|c|c|c|c|c|c|}
\hline \multirow[t]{3}{*}{ Variable } & \multirow[t]{3}{*}{ Category } & \multicolumn{5}{|c|}{$\begin{array}{l}\text { Current smoker who answered the willingness to } \\
\text { quit question in student cohort ( } \mathrm{N}=666)\end{array}$} & \multicolumn{5}{|c|}{$\begin{array}{c}\text { Current smoker who answered the willingness to } \\
\text { quit question in staff cohort ( } N=1184)\end{array}$} & \multicolumn{5}{|c|}{$\begin{array}{l}\text { Current smoker who answered the willingness to } \\
\text { quit question in both cohorts }(\mathrm{N}=1850)\end{array}$} \\
\hline & & \multirow{2}{*}{$\begin{array}{l}\mathrm{N}=552 \\
\text { in the } \\
\text { model }\end{array}$} & \multirow[t]{2}{*}{ AOR } & \multicolumn{2}{|c|}{$95 \%$ CI } & \multirow[t]{2}{*}{$p$} & \multirow{2}{*}{$\begin{array}{c}\mathbf{N}=984 \\
\text { in the } \\
\text { model }\end{array}$} & \multirow[t]{2}{*}{ AOR } & \multicolumn{2}{|c|}{$95 \%$ CI } & \multirow[t]{2}{*}{$p$} & \multirow{2}{*}{$\begin{array}{c}N=1536 \\
\text { in the } \\
\text { model }\end{array}$} & \multirow[t]{2}{*}{$\mathrm{AOR}$} & \multicolumn{2}{|c|}{$95 \%$ CI } & \multirow[t]{2}{*}{$p$} \\
\hline & & & & Lower & Upper & & & & Lower & Upper & & & & Lower & Upper & \\
\hline \multirow[t]{2}{*}{ Gender } & Female & 392 & 1 & & & & 726 & 1 & & & & 1118 & 1 & & & \\
\hline & Male & 160 & 1.004 & 0.650 & 1.552 & 0.985 & 258 & 1.083 & 0.759 & 1.545 & 0.662 & 418 & 1.043 & 0.796 & 1.368 & 0.759 \\
\hline \multirow[t]{5}{*}{ Age (years) } & $35-44$ & 16 & 1 & & & & 257 & 1 & & & & 273 & 1 & & & \\
\hline & $\leq 25$ & 499 & 0.686 & 0.183 & 2.576 & 0.577 & 163 & 0.914 & 0.581 & 1.438 & 0.697 & 662 & 0.860 & 0.571 & 1.295 & 0.470 \\
\hline & $26-34$ & 32 & 0.660 & 0.146 & 2.975 & 0.588 & 267 & 0.827 & 0.559 & 1.222 & 0.340 & 299 & 0.821 & 0.565 & 1.193 & 0.301 \\
\hline & $45-54$ & 5 & $\mathrm{NC}$ & $\mathrm{NC}$ & $\mathrm{NC}$ & $\mathrm{NC}$ & 207 & 0.802 & 0.530 & 1.212 & 0.294 & 212 & 0.835 & 0.557 & 1.251 & 0.381 \\
\hline & $\geq 55$ & 0 & & & & & 90 & 0.773 & 0.450 & 1.330 & 0.353 & 90 & 0.776 & 0.454 & 1.324 & 0.351 \\
\hline \multirow{3}{*}{$\begin{array}{l}\text { Information about } \\
\text { smoking }\end{array}$} & No & 92 & & & & & 204 & 1 & & & & 296 & 1 & & & \\
\hline & Yes & 445 & 1.251 & 0.748 & 2.092 & 0.393 & 734 & 1.187 & 0.826 & 1.704 & 0.354 & 1179 & 1.219 & 0.910 & 1.632 & 0.184 \\
\hline & Refusal & 15 & 0.676 & 0.200 & 2.283 & 0.528 & 46 & 1.067 & 0.521 & 2.184 & 0.860 & 61 & 0.957 & 0.519 & 1.764 & 0.888 \\
\hline \multirow[t]{8}{*}{ Occupation } & Administrative & 0 & & & & & 143 & 1 & & & & 143 & 1 & & & \\
\hline & Technical/security & 0 & & & & & 50 & 0.844 & 0.405 & 1.761 & 0.652 & 50 & 0.857 & 0.417 & 1.762 & 0.674 \\
\hline & Logistical & 0 & & & & & 53 & 1.330 & 0.621 & 2.845 & 0.463 & 53 & 1.413 & 0.671 & 2.974 & 0.363 \\
\hline & Scientific & 0 & & & & & 18 & 1.474 & 0.484 & 4.489 & 0.495 & 18 & 1.444 & 0.476 & 4.385 & 0.517 \\
\hline & Medical & 0 & & & & & 115 & 0.680 & 0.374 & 1.236 & 0.206 & 115 & 0.688 & 0.383 & 1.239 & 0.213 \\
\hline & Paramedical & 0 & & & & & 605 & 0.884 & 0.578 & 1.353 & 0.571 & 605 & 0.870 & 0.572 & 1.325 & 0.517 \\
\hline & Medical student & 344 & 1 & & & & 0 & & & & & 344 & 0.641 & 0.372 & 1.104 & 0.109 \\
\hline & Paramedical student & 208 & 1.690 & 1.105 & 2.584 & 0.015 & 0 & & & & & 208 & 1.134 & 0.656 & 1.959 & 0.652 \\
\hline \multirow[t]{2}{*}{ Feel symptoms } & No & 381 & 1 & & & & 685 & 1 & & & & 1066 & 1 & & & \\
\hline & Yes & 171 & 1.792 & 1.169 & 2.747 & 0.007 & 299 & 4.094 & 2.907 & 5.766 & $<10^{-4}$ & 470 & 2.968 & 2.288 & 3.849 & $<10^{-4}$ \\
\hline \multirow[t]{4}{*}{ Quit attempts } & 0 & 244 & 1 & & & & 292 & 1.000 & & & & 536 & 1 & & & \\
\hline & 1 & 133 & 2.820 & 1.774 & 4.482 & $<10^{-4}$ & 240 & 2.259 & 1.548 & 3.295 & $<10^{-4}$ & 373 & 2.470 & 1.849 & 3.301 & $<10^{-4}$ \\
\hline & 2 & 80 & 3.368 & 1.941 & 5.841 & $<10^{-4}$ & 172 & 2.494 & 1.642 & 3.787 & $<10^{-4}$ & 252 & 2.812 & 2.024 & 3.908 & $<10^{-4}$ \\
\hline & $>2$ & 95 & 9.347 & 5.136 & 17.010 & $<10^{-4}$ & 280 & 4.882 & 3.296 & 7.230 & $<10^{-4}$ & 375 & 5.885 & 4.275 & 8.101 & $<10^{-4}$ \\
\hline \multirow{3}{*}{$\begin{array}{l}\text { Cigarettes smoked/ } \\
\text { day }\end{array}$} & $<10$ & 442 & 1 & & & & 649 & 1 & & & & 1091 & 1 & & & \\
\hline & $10-20$ & 99 & 0.960 & 0.581 & 1.585 & 0.873 & 292 & 0.934 & 0.677 & 1.290 & 0.680 & 391 & 0.932 & 0.713 & 1.218 & 0.606 \\
\hline & $>20$ & 11 & 0.065 & 0.007 & 0.578 & 0.014 & 43 & 0.518 & 0.253 & 1.060 & 0.072 & 54 & 0.393 & 0.209 & 0.741 & 0.004 \\
\hline \multirow[t]{2}{*}{ Overnight work } & No & 0 & & & & & 748 & 1 & & & & 748 & 1 & & & \\
\hline & Yes & 0 & & & & & 236 & 1.433 & 1.013 & 2.026 & 0.042 & 236 & 1.430 & 1.013 & 2.018 & 0.042 \\
\hline
\end{tabular}

AOR: adjusted odds ratio. NC: Not calculated (low effective in the category). 
willingness to quit were in number of previous quit attempts in an increasing way: once $(\mathrm{AOR}=2.470$; 95\% CI: $1.849-3.301)$ to more than twice $(\mathrm{AOR}=5.885 ; 95 \% \mathrm{CI}: 4.275-8.101)$ for more than twice), and feeling tobacco-related symptoms $(\mathrm{AOR}=2.968 ;$ 95\% CI: 2.288-3.849). Other variables were either not or marginally significant.

\section{DISCUSSION}

This study reports a snapshot of smoking behavior and factors associated with tobacco use, among staff and students in the University Hospital of Lyon. Overall, the prevalence rate of smoking was $25 \%$ (i.e. $26 \%$ in students and $25 \%$ in staff). Various characteristics affecting smoking status were identified. Among the youngest age categories there were many current smokers. Among logistical staff and night workers there were more current smokers. There were fewer current smokers in medical students, relative to paramedical students.

After a decreasing smoking prevalence in the past decades in France, an increase between 2005 and 2010 was observed. Stagnation between 2010 and 2016 was followed by a smoking prevalence decrease from $29 \%$ of daily current smokers in 2016 to $27 \%$ in $2017^{10}$. A government action plan against tobacco smoking could explain this evolution. Various steps built this plan, notably increasing tobacco prices and implementation of neutral tobacco packaging.

Many initiatives were taken recently to reduce smoking prevalence in the general population. In France, the government created a national plan to fight tobacco smoking named Programme National de Lutte Contre le Tabac ${ }^{11}$. Among student populations, this program specifically establishes a 'student ambassador' who is entrusted with the role of promoting smoking cessation among other students. In addition, programs promoting smokefree hospitals are growing, such as the 'Keeping your hospital property smoke-free' from the Joint Commission in the United States ${ }^{12}$. In France, a similar approach is promoted by various academics such as RESPADD, an associative network helping hospital staff and students to stop smoking ${ }^{13}$.

The last assessment of the smoking prevalence among the French general population found an estimate of $32 \%$ current smokers among adults with $27 \%$ daily users; and $32 \%$ of current smokers in the 18-24 years group ${ }^{10}$. Overall, we observed a lower prevalence rate of current smoking in our study ( $25 \%$ of hospital staff and students) than in the general population. We also found an overall prevalence of $33 \%$ current smokers in the 20-25 years group. This represents the only age range in which smoking prevalence in our hospital was higher than in the general population. However, it follows the downward trend observed in France with a smoking prevalence in the $18-24$ years group in 2016 of $37 \%{ }^{10}$. In our cohort, $28 \%$ among men and $24 \%$ among women were current smokers. This difference is similar to the overall population, with $30 \%$ in men and $24 \%$ in women ${ }^{10}$. However, we found no gender difference among the student cohort: $26 \%$ for both women and men.

There is a high variability in the literature about smoking prevalence among hospital staff and students. In the Pianori et al. ${ }^{3}$ cross-sectional study consisting of three surveys in 2006, 2011 and 2015 , they found an overall smoking prevalence of $35 \%$ in the hospital staff whereas there were $20 \%$ current smokers in the general Italian population ${ }^{3}$. They also found a higher rate of current smokers among technical workers, but without a statistically significant difference. Medical students need adequate preparation, but these topics are rarely approached in traditional curricula despite their importance. In Italy, the knowledge of nicotine dependence and its treatment in medical students is generally low and recent work reports the effectiveness of an online course at increasing knowledge of the harmful effects of tobacco, tobacco dependence and treatments for dependence ${ }^{14,15}$. In 2004 in Greece, Vagropoulos et al. ${ }^{4}$ measured by questionnaire the prevalence of smoking in a public Greek hospital: $50 \%$ were current smokers which is higher than general population (38\%). The lowest prevalence was found in the medical staff ${ }^{4}$. By contrast, in the Metropolitan hospital in Melbourne, a large Australian public hospital, they found in 2012 a lower smoking prevalence among staff than in the general population ( $7 \%$ and $16 \%$, respectively $)^{6}$.

It is well known that socioeconomic factors influence smoking status. 'blue-collar' workers are described in American studies to be more likely to smoke than 'white collar' workers ${ }^{16-18}$. Our study supports the importance of socioeconomic status 
in smoking behavior and this could give argument to tailor smoking prevention programs to some occupational categories. We also highlight the importance of working conditions on smoking status. Indeed, overnight work in our study is associated with a greater proportion of current smokers. Different studies support this result. For example, Van Amelsvoort et al. ${ }^{19}$ conducted a longitudinal study in 2006 showing a 1.46 odds ratio of being current smoker when people work on shift compared to daytime workers ${ }^{19,20}$. Educational level and occupational categories are important predictors of smoking. Our findings are consistent with previous studies: medical staff and students are less likely to smoke than other healthcare workers ${ }^{21,22}$. More efforts to explain those differences, identify barriers to quitting and implement targeted interventions to help health professional to quit smoking are essential.

The prevalence of medical staff and students selfestimate as being well informed about tobacco is $84 \%$ and $87 \%$, respectively. In France, the ' 2015 cancer barometer' revealed similar rates with $89 \%$ to $95 \%$ of participants considering themselves as being well informed about tobacco risks ${ }^{23}$.

Fighting against tobacco smoking in medical and paramedical occupations is crucial. Beyond their own physical health, various studies suggest smokers are less able to give advice to help patients to give up smoking. For example, in an Italian and American study, it was observed that current smokers among medical students were less likely to offer smoking cessation counseling or treatment to patients ${ }^{24}$. Similar results were found in a Lebanese study which reported 182 medical student questionnaires about their smoking status and attitude toward smoking ${ }^{7}$. We could therefore obtain great public health benefit in future targeted interventions in this population.

In multivariate analysis, the variables associated with willingness to quit were: feeling symptoms, and number of quit attempts. Our finding that the number of previous quit attempts is positively related with intention to quit is in agreement with the literature ${ }^{25,26}$. Unsuccessful quit attempts do not decrease motivation for smoking cessation. Therefore, this population remains a particular target group for health promotion. Feeling symptoms is probably the main factor associated with willingness to quit found in the literature ${ }^{27}$. Smoking cessation before health consequences occur represents the main public health preventive measure. Approximately half of smoker participants reported a willingness to quit smoking. In 2018, in a large survey among Indian smokers Reddy et al. ${ }^{28}$ found the same prevalence of willingness to quit, while in the 2017 overall French population, there were 57\% willing to quit compared with $60 \%$ in $2014^{10,29}$.

\section{Strengths and limitations}

This study has strengths to highlight. First, the participation rate and the sample size were particularly high: we collected 9712 questionnaires corresponding to a $41 \%$ participation rate in the student cohort and $52 \%$ in the staff cohort ${ }^{30}$. To our knowledge this study belongs to the biggest studies about smoking prevalence among hospital staff and students worldwide: by way of illustration, a meta-analysis about tobacco use prevalence among healthcare workers reported in 2019 a median sample size of $375^{31}$. Second, we included both students and staff while many similar studies did not. Here we offer an overview of broad socioeconomic and age ranges, allowing informative comparisons.

One important limitation is the single day questionnaire, with binary responses without quantitative variables. Many eligible staff and students were not included, resulting in a loss of precision in the collected information. Besides, multiplicity of statistical analyses may have had an impact on type I and II errors. Otherwise, missing values were frequent but are reported as such. Finally, the self-report of all variables including smoking status represents a strong limitation of this kind of study. In addition, current smokers may have been less likely to answer the questionnaire, leading to an underestimate of current smoker prevalence.

\section{CONCLUSIONS}

A lower prevalence of current smoking is observed in our populations of students and staff than in the general population. However, those rates remain high and encourage us to implement a strong tobacco control policy before repeating this study in 5 years to evaluate it. Prevention looks especially important in students because of their future impact on smoking 
prevention among patients and their young age. The smoking prevention campaigns should be oriented toward most affected categories by the tobacco epidemic, such as lower socioeconomic populations, young people, night workers or paramedical students, who present a high rate of current smoking in our study. Studying beliefs about tobacco smoking could be informative for their determining role in the smoking cessation process.

Feeling symptoms and number of previous quit attempts are main variables associated with willingness to quit. Our study could help to target former and current smokers eligible for lung cancer CT scan screening. The planned second step of the study to be conducted in a five-year timeframe will integrate this objective.

\section{REFERENCE}

1. Forouzanfar MH, Afshin A, Alexander LT, et al. Global, regional, and national comparative risk assessment of 79 behavioural, environmental and occupational, and metabolic risks or clusters of risks, 1990-2015: a systematic analysis for the Global Burden of Disease Study 2015. The Lancet. 2016;388(10053):1659-724. doi:10.1016/S0140-6736(16)31679-8

2. Bonaldi C, Andriantafika F, Chyderiotis S, et al. Smokingattributable mortality in France. Latest estimates and trend, 2000-2013. Bull Epidémiol Hebd. 2016;(3031):528-540. Accessed May 18, 2021. https://www. researchgate.net/publication/311789658_Smokingattributable_mortality_in_France_Latest_estimates_and_ trend_2000-2013_in_French

3. Pianori D, Gili A, Masanotti G. Changing the smoking habit: prevalence, knowledge and attitudes among Umbrian hospital healthcare professionals. J Prev Med Hyg. 2017;58(1):E72-E78. Accessed May 18, 2021. https://www.ncbi.nlm.nih.gov/pmc/articles/ PMC5432782/pdf/2421-4248-58-E72.pdf

4. Vagropoulos I, Tsilchorozidou T, Tsinopoulos G, et al. Smoking habits among the hospital staff of a General Hospital in northern Greece: a long way for smoke-free hospitals. Monaldi Arch Chest Dis. 2006;65(3):160-164. doi:10.4081/monaldi.2006.562

5. Lewis KE, Shin D, Davies G. Smoking habits and attitudes toward tobacco bans among United Kingdom hospital staff and students. Int J Tuberc Lung Dis. 2011;15(8):11221126. doi:10.5588/ijtld.10.0783

6. Rahman MA, Wilson A, Sanders R, et al. Smoking behavior among patients and staff: a snapshot from a major metropolitan hospital in Melbourne, Australia. Int J Gen Med. 2014;7:79. doi:10.2147/IJGM.S54230

7. Chidiac A, Tamim H, Kanso M, et al. Smoking among Lebanese medical students: Prevalence and attitudes. Ann Thorac Med. 2016;11(3):183. doi:10.4103/1817-1737.185757

8. Von Elm E, Altman DG, Egger M, et al. The Strengthening the Reporting of Observational Studies in Epidemiology (STROBE) statement: guidelines for reporting observational studies. J Clin Epidemiol. 2008;61(4):344349. doi:10.1016/j.jclinepi.2007.11.008

9. Couraud S, Zalcman G, Milleron B, et al. Lung cancer in never smokers - A review. Eur J Cancer. 2012;48(9):1299-1311. doi:10.1016/j.ejca.2012.03.007

10. Pasquereau A, Andler A, Guignard G, et al. Tobacco consumption in France: preliminary results from the 2017 health barometer. Article in French. Bull Epidémiol Hebd. 2018;(14-15):265-273. Accessed May 18, 2021. http:// beh.santepubliquefrance.fr/beh/2018/14-15/2018_1415_1.html

11. Republique Francaise. PROGRAMME NATIONAL DE LUTTE CONTRE LE TABAC 2018-2022. Accessed November 12, 2020. https://solidarites-sante.gouv.fr/ IMG/pdf/180702-pnlt_def.pdf

12. The Joint Commission. Keeping Your Hospital Property Smoke-Free: Successful Strategies for Effective Policy Enforcement and Maintenance. The Joint Commission, Henry Ford Health System; 2011. Accessed November 12, 2020. https://www.jointcommission.org/-/media/ deprecated-unorganized/imported-assets/tjc/systemfolders/topics-library/smoke_free_brochure2pdf.pdf?db =web\&hash=E237FA9881AD54F78442DC3F262C2FD7

13. The RESPADD Prevention and Addiction network. Respadd. Accessed November 12, 2020. https://www. respadd.org/qui-sommes-nous/

14. Grassi MC, Chiamulera C, Baraldo M, et al. Cigarette Smoking Knowledge and Perceptions Among Students in Four Italian Medical Schools. Nicotine Tob Res. 2012;14(9):1065-1072. doi:10.1093/ntr/ntr330

15. Grassi MC, Sansone A, Basili S, Ferketich AK. Knowledge of nicotine dependence and treatment in clinical practice improved after an e-learning course among medical students. Clin Ter. 2019;170(4):e252-e257. doi:10.7417/CT.2019.2142

16. Nelson DE, Emont SL, Brackbill RM, et al. Cigarette smoking prevalence by occupation in the United States. A comparison between 1978 to 1980 and 1987 to 1990 . J Occup Med. 1994;36(5):516-525.

17. Giovino G, Pederson L. The Prevalence of Selected Cigarette Smoking Behaviors by Occupational Class in the United States. In: Work, Smoking, and Health, a NIOSH Scientific Workshop; June 15-16, 2000; Washington, DC. Accessed November 15, 2020. https://www.cdc.gov/ niosh/docs/2002-148/pdfs/2002-148.pdf

18. Sorensen G, Emmons K, Stoddard M, et al. Do social influences contribute to occupational differences in quitting smoking and attitudes toward quitting? Am J Health Promot. 2002;16(3):135-141. doi:10.4278/0890-1171-16.3.135

19. Van Amelsvoort LGPM, Jansen NWH, Kant I. Smoking 
among Shift Workers: More Than a Confounding Factor. Chronobiol Int. 2006;23(6):1105-1113. doi:10.1080/07420520601089539

20. Buchvold HV, Pallesen S, Øyane NMF, et al. Associations between night work and BMI, alcohol, smoking, caffeine and exercise - a cross-sectional study. BMC Public Health. 2015;15:1112. doi:10.1186/s12889-015-2470-2

21. Syamlal G, Mazurek JM, Storey E, et al. Cigarette Smoking Prevalence Among Adults Working in the Health Care and Social Assistance Sector, 2008 to 2012. J Occup Environ Med. 2015;57(10):1107-1112. doi:10.1097/JOM.0000000000000529

22. Sarna L, Bialous SA, Nandy K, et al. Changes in Smoking Prevalences Among Health Care Professionals From 2003 to 2010-2011. JAMA. 2014;311(2):197. doi:10.1001/jama.2013.284871

23. Pasquereau A, Deutsch A, Richard JB, et al. Tabac et cancer. Perception des risques en 2015 et évolutions récentes. Baromètre Cancer 2015. Santé Publique France; 2019:18.

24. Armstrong GW, Veronese G, George PF, et al. Assessment of Tobacco Habits, Attitudes, and Education Among Medical Students in the United States and Italy: A Crosssectional Survey. J Prev Med Pub Health. 2017;50(3):177187. doi:10.3961/jpmph.15.061

25. Marques-Vidal P, Melich-Cerveira J, Paccaud F, et al. Prevalence and factors associated with difficulty and intention to quit smoking in Switzerland. BMC Public Health. 2011;11(1):227. doi:10.1186/1471-2458-11-227

26. Feng G, Jiang Y, Li Q, et al. Individual-level factors associated with intentions to quit smoking among adult smokers in six cities of China: findings from the ITC China Survey. Tob Control. 2010;19(Suppl 2):i6-i11. doi:10.1136/tc.2010.037093

27. McCaul KD, Hockemeyer JR, Johnson RJ, et al. Motivation to quit using cigarettes: A review. Addict Behav. 2006;31(1):42-56. doi:10.1016/j.addbeh.2005.04.004

28. Reddy M, Kanungo S, Naik B, et al. Willingness to quit tobacco smoking and its correlates among Indian smokers - Findings from Global Adult Tobacco Survey India, 2009-2010. J Fam Med Prim Care. 2018;7(6):1353. doi:10.4103/jfmpc.jfmpc_169_18

29. Guignard R, Nguyen-Thanh V, Richard J-B, et al. Evolution of tobacco smoking in France: results from the health barometer 2014. Article in French. 2015;8(17-18). Accessed May 18, 2021. http://beh.santepubliquefrance. fr/beh/2015/17-18/pdf/2015_17-18.pdf

30. Brown EM, Olson LT, Farrelly MC, et al. Comparing Response Rates, Costs, and Tobacco-Related Outcomes Across Phone, Mail, and Online Surveys. Surv Pract. 2018;11(2):4406. doi:10.29115/SP-2018-0029

31. Nilan K, McKeever TM, McNeill A, et al. Prevalence of tobacco use in healthcare workers: A systematic review and meta-analysis. PLoS ONE. 2019;14(7):e0220168. doi:10.1371/journal.pone.0220168
CONFLICTS OF INTEREST

The authors have each completed and submitted an ICMJE form for disclosure of potential conflicts of interest. The authors declare that they have no competing interests, financial or otherwise, related to the current work. B. Rolland reports grants from Abbvie, Gilead, and Camurus, consulting fees from Abbvie, Gilead, Camurus, Pileje, Accord Health, Indivior, and Zentiva, honorary payments from Abbvie, Gilead, Recordati, Ethypharm, HAC Pharma, and Shire, and other payment from Camurus, outside the submitted work. M. Darrason reports honorary payment from Bristol Meyers Squibb, CCA congress colloquia, outside the submitted work.

\section{FUNDING}

The research was sponsored by Hospices Civils de Lyon under the reference 69HCL17_0481.

\section{ETHICAL APPROVAL AND INFORMED CONSENT}

This study was approved by the Sud-Ouest et Outre Mer III ethics committee (Comité de Protection des Personnes) under the reference 2017A02168-45 on 25 October 2017. It was declared to the local correspondent from French Informatics and Freedom Agency under the reference MR0003-17-153. It was registered in clinicaltrials.gov under the reference NCT03268980.

\section{DATA AVAILABILITY}

The data supporting this research can be found in the Supplementary file.

\section{PROVENANCE AND PEER REVIEW}

Not commissioned; externally peer reviewed. 